\title{
edoc
}

Institutional Repository of the University of Basel

University Library

Schoenbeinstrasse 18-20

CH-4056 Basel, Switzerland

http://edoc.unibas.ch/

Year: 2011

\section{Mechanisms for complexity in the brain : generating the insect central complex}

Boyan, G. S. and Reichert, H.

Posted at edoc, University of Basel

Official URL: http://edoc.unibas.ch/dok/A5844180

Originally published as:

Boyan, G. S. and Reichert, H.. (2011) Mechanisms for complexity in the brain : generating the insect central complex. Trends in neurosciences, Vol. 34. S. 247-257. 
Mechanisms for Complexity in the Brain: Generating the Insect Central Complex

\section{George S. Boyan' ${ }^{1}$ and Heinrich Reichert²}

${ }^{1}$ George S. Boyan is at the Biocenter, University of Munich, Grosshadernerstr. 2, 82152 Martinsried, Germany

${ }^{2}$ Heinrich Reichert is at the Biozentrum, University of Basel, Klingelbergstrasse 50, 4056 Basel, Switzerland

Corresponding author: Reichert, H. (heinrich.reichert@unibas.ch).

Keywords: Brain, Central complex, Development, Progenitors, Lineages, Neural stem cells, Proliferation, Drosophila

Running title: Mechanisms for Complexity in the Brain 


\section{Abstract}

The central complex of the insect brain is a remarkably miniaturized yet highly complex multimodal information processing network. Recent work on central complex development in Drosophila and grasshopper reveals that the cells comprising its complex circuitry are generated by a surprisingly small number of primary progenitors. Among these, four identified neural stem cells generate a large number of neurons though a novel mode of neurogenesis that involves self-renewing intermediate progenitor cells. Interestingly, a comparable mode of amplification of proliferation also operates in the developing mammalian cortex, and may be a general strategy for increasing brain size and complexity. While this type of proliferation generates a large number of progeny, it is also prone to dysregulation, resulting in brain tumors. Thus, furthering our knowledge of the development of the central complex is likely to be valuable not only for understanding brain complexity, but also may have important implications for identifying developmental pathways that go awry during tumor formation. 


\section{Introduction}

The insect brain is a highly complex structure composed of hundreds of thousands of neurons that are interconnected in numerous exquisitely organized neuropile assemblies such as the optic lobes, mushroom bodies, antennal lobes and central complex [1](Glossary). The central complex, located in the midline of the protocerebrum (forebrain), comprises thousands of neurons representing some 50 neural types arranged in a modular neuropile neuroarchitecture whose basic elements are remarkably conserved across insect species [1-9]. In insects, such as the grasshopper Schistocerca and the fruitfly Drosophila, the central complex comprises five major modules: the protocerebral bridge (PB), central body (CB), ellipsoid body (EB), noduli (N) and lateral accessory lobes (LAL) (see Box 1 Fig. Ia, b, c). The central complex is involved in multimodal information processing and visual memory storage as well as in coordinating motor behaviors such as walking, flying and stridulation [10-18]. Accordingly, mutant analyses in Drosophila reveal that malformed or missing modules have profound effects on coordinated locomotory behavior $[11,12,19]$.

The intricate neural circuitry of the central complex is generated during development. Insight into the cellular and molecular mechanisms that operate during central complex development has come from both the grasshopper, an advantageous cellular model system, and the fruitfly, an excellent genetic model system. In both of these insect model systems, the large numbers of neural elements that make up the central complex are generated by a small set of identified stem cell-like primary progenitors [20-24]. Some of these primary progenitors have been shown to generate astonishingly large lineages of neural progeny through a novel form of neurogenesis involving amplification of proliferation through selfrenewing intermediate progenitors [25-29]. This review focuses on the remarkable cellular and molecular mechanisms involved in generating the large cell numbers required to initiate and complete central complex development during a restricted time window. The parallels revealed in insect brain development as compared to mammalian brain 
development suggest that amplifying intermediate progenitor cells may represent a general and evolutionarily conserved mechanism for producing the large neural cell numbers and diverse cell types necessary for complex brain circuitry.

\section{Neural stem cells and lineage-specific development of the central complex}

In the grasshopper and Drosophila, the neurons of the central brain, defined as the supraesophageal ganglion without the optic lobes [30], derive from approximately 100 bilaterally symmetrical pairs of primary progenitors with neural stem cell properties (often referred to as neuroblasts for historical reasons), each of which has been individually identified [31-38]. Neural stem cells (NSCs) are primary progenitor cells that give rise to neural lineages comprising differentiated neurons or glial cells (for a review, see [39]). A number of studies indicate that each of these developing NSCs acquires an intrinsic capacity for neuronal proliferation in a cell autonomous manner and generates a specific lineage of neural progeny which is nearly invariant and unique (for a review, see [40]). This implies that each NSC acquires a specific identity which determines the number and types of neural progeny it generates. This specification of NSCs has been shown to occur via a combination of positional information, temporal cues, and combinatorial cues provided by the suite of developmental control genes expressed by each progenitor [28,4144].

As is the case for the other neuropile structures in the central brain, the neurons of the central complex are generated through the proliferative activity of NSCs. Surprisingly, recent investigations indicate that a relatively small number of the central brain's NSCs generate the remarkably large number of neurons that make up the central complex. This is noteworthy since the central complex commands a high proportion of the neurons found in the brain $[1,21,45]$, a fact which documents that there has been a major evolutionary investment towards generating its intricate neuroarchitecture [2,7,46]. Further, developmental constraints require that this complex neuroarchitecture be built within a 
very limited time frame; during mid- to late-embryogenesis in organisms that develop directly (grasshopper) [38], and during the larva to adult transition for organisms that develop indirectly (Drosophila) [47-49]. This raises the question of how such a large number of neurons can be generated by such a small number of NSCs during such a short developmental time period.

An identified subset of the NSCs in the brain contributes neural elements to the developing central complex in grasshopper and Drosophila (Box 1). Cellular analyses show that four of these identified NSCs located bilaterally at the midline of each protocerebral brain hemisphere play a key role in central complex development in that their progeny establish the basic columnar organization of this neuropile (Box 1 Fig. Id). In the grasshopper (Fig. 1a), these NSCs, termed W, X, Y, Z, each generate a lineage-related suite of neurons (Fig. 1b-e) which contribute fibers to substructures of the central complex via the so-called w, x, y, z brain tracts (also see Box 1 Fig. Ib) [34,38]. Genetic analysis reveals that a similar set of four NSCs is found in the developing brain of Drosophila, and each of these four NSCs also generates a specific lineage-related cluster of neurons which contribute fibers to the developing central complex (Fig. 1g) [21-24].

In Drosophila, these NSCs as well as the lineages of neurons that they generate can be labeled specifically using clonal molecular genetic labeling techniques such as mosaic analysis with a repressible cell marker (MARCM) [50] (Fig. 1h). This type of lineagespecific genetic labeling shows that the four lineages comprise the numerous columnar or small field neurons that project to, innervate and interconnect the protocerebral bridge, fan-shaped body, ellipsoid body and noduli of the central complex. In both grasshopper (Fig. 1c-e) and Drosophila (Fig. 1h), the cellular organization of each of these NSC-derived lineages is relatively stereotyped, with the large NSC located near the brain hemisphere's surface and its progeny extending in a clustered orderly array towards the central neuropile such that the relative location of the cells within the cluster corresponds to their birthdate. This results in a specific temporal topology of the lineage (Fig. 1f). This temporal 

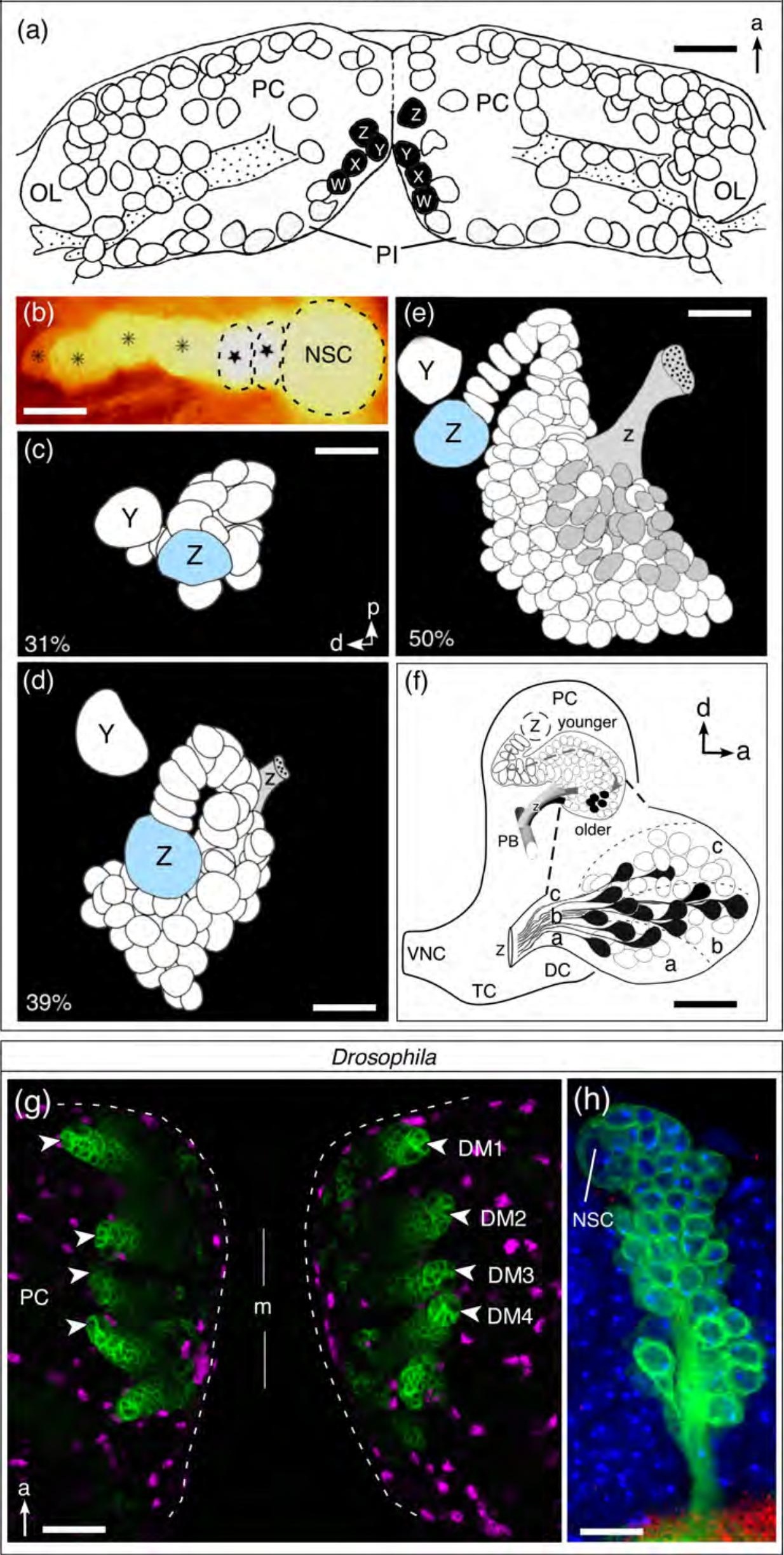
topology is reflected in the formation of central complex circuitry in that the neurites of later born neurons often project their processes along those of early born neurons to form fascicle arrays that subsequently undergo more complex morphogenesis.

\section{Novel forms of neurogenesis in central complex development}

Recent studies demonstrate that two types of NSC-derived lineages exist in the developing central brain [25-29]. Most of the brain's NSCs give rise to so-called Type I lineages, for example those which in the grasshopper are associated with the diffuse neuropile or the mushroom bodies (Fig. 2a)[29]. In contrast, a small subset of the NSCs gives rise to socalled Type II lineages such as those associated with the $\mathrm{W}, \mathrm{X}, \mathrm{Y}, \mathrm{Z}$ system of the grasshopper central complex (Fig. 2b)[29]. These lineages contain 4 to 5 times more cells as compared with most Type I lineages at the same stage of development (Fig. 2c). Accordingly the four w, x, y, z Type II lineages occupy an over-proportionally large volume of each brain hemisphere (see Fig. 1f) [29,38].

These striking differences between Type I and Type II lineages are also manifest in the developing brain of Drosophila [25-28]. Here, most Type I NSCs generate lineages comprising on average 100 - 120 adult-specific cells (Fig. 2d). In contrast, a total of 8 Type II NSCs, including the four mideline-associated NSCs that generate small field neurons of the central complex, produce lineages that have an average value of 450 (range 370-580) cells and thus are considerably larger than the lineages contributing to other protocerebral structures in each brain hemisphere (Fig. 2e) [22,25].

In Drosophila and in the grasshopper, a further interesting feature of some of the Type II lineages has been discovered [22,29]. These lineages not only comprise a large set of neurons, they also contain glial cells implying that Type II NSCs are multipotent primary progenitors that can generate both neurons and glia (Fig. 2f, g, h).

In both insect model systems, the neural cells of the Type II central complex lineages are generated during a restricted developmental period. All of the neurons are present by 


\section{grasshopper}

(a) Type I lineages * $* * * 200$
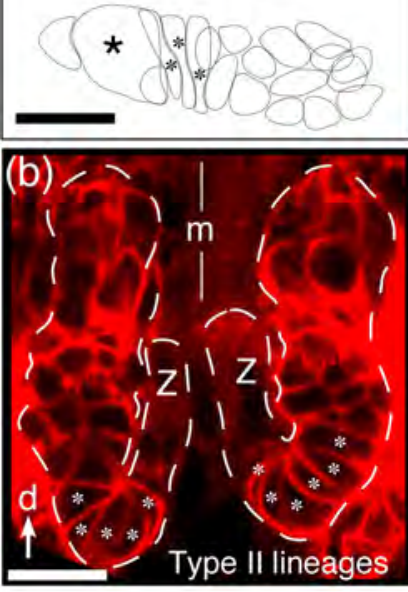

(c)

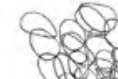

(1)

sin

(

120

on

ene

(ex)e 70

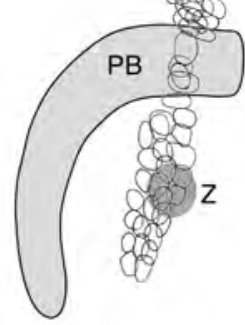

Type II lineages

\section{Drosophila}

(d)

Type I lineages (e)

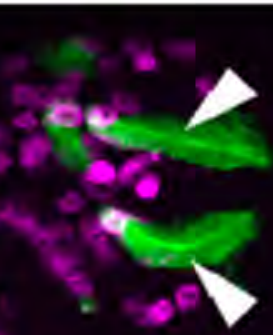

(f)

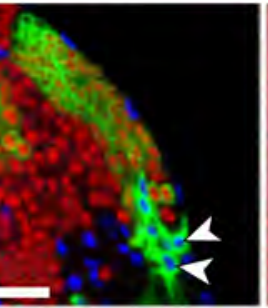

GFP

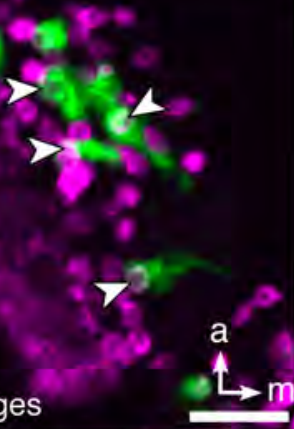

Type II lineages

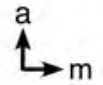
w

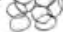

PB

J

$m$ 
mid-embryogenesis in the grasshopper $[34,38]$ and by early metamorphosis in Drosophila [25]. This contrasts with some of the Type I lineages where neurons are generated during much longer developmental periods. For example, the Type I NSCs that generate the intrinsic cells of the mushroom body proliferate throughout development and, in some insects which develop directly, continue to proliferate even into adulthood [51]. This implies that Type II lineages can only attain their extraordinary size in the restricted time available if additional, Type II-specific mechanisms for generating progeny are available. One such novel mechanism for increasing neural proliferation has recently been discovered in the NSCs that contribute to central complex development. These Type II NSCs amplify neural proliferation through the generation of self-renewing intermediate neural progenitor cells (INPs) that act as transit amplifying cells.

\section{Amplification of neural stem cell proliferation through intermediate progenitor cells}

In the "classical" mode of neurogenesis that operates in Type I lineages, the NSCs as primary progenitors divide asymmetrically in a stem cell mode to self-renew and generate a smaller daughter cell called a ganglion mother cell (GMC). The GMC is a non selfrenewing secondary progenitor that undergoes a single terminal division to produce two postmitotic progeny, neurons or glial cells $[28,41,52,53]$. In Type II lineages, the NSCs as primary progenitors also divide asymmetrically in a stem cell mode to self-renew and generate a smaller daughter cell, however, this daughter cell is a self-renewing intermediate neural progenitor (INP) which has features characteristic of transit amplifying cells [25-27]. Since each INP has the capacity to undergo a limited number of (up to 10) divisions in which it self-renews, INPs have the potential to amplify neural cell numbers markedly in the lineages in which they occur [54].

In the grasshopper, a group of mitotically active INPs is found in each of the (Type II) $\mathrm{w}, \mathrm{x}, \mathrm{y}, \mathrm{z}$ lineages of the central complex at mid-embryogenesis (Fig. 3a, b) [29]. These 
INPs can be identified based on criteria such as cell shape and location, spindle orientation, immunoreactivity and mode of division (self-renewal) (Fig. 3c). Each INP may give rise, via multiple rounds of self-renewal, to a discrete subset of neural progeny within the lineage. The INPs from a given lineage appear to be phase-locked with respect to the cell cycle, remain in place for almost a fifth of total embryonic time, and cease mitotic activity simultaneously during late embryogenesis [29].

In Drosophila, the corresponding Type II NSCs as well as the INPs generated by these NSCs can be labeled and analysed molecularly using combinations of clonal genetic and immunocytochemical molecular labeling techniques (Fig. 3d-h) [22,23,25-27,54]. The INPs in each of these lineages are arranged as a clustered group of mitotically active cells in the general vicinity of their parent NSC. Moreover, they can be unequivocally distinguished from the (non self-renewing) GMCs in the lineage by the polarized expression of the coiled-coil protein Miranda during mitosis [28,53]. Another distinguishing fact is that INPs divide repeatedly to produce multicellular neuronal clones composed of more than two postmitotic cells, which can be labeled with MARCM techniques [25]. Interestingly, newly generated INPs represent an immature cell type that is arrested in the G2 phase of the cell cycle [26,27]. Before re-entering the cell cycle, these immature INPs must undergo a maturation process during which they acquire and actively maintain their restricted proliferative potential (see Box 2 Fig. IIe) [54].

\section{Molecular mechanisms underlying amplification}

What are the molecular mechanisms that underlie the amplification of neurogenesis in Type II lineages? All central brain NSCs undergo asymmetric cell divisions in which they self-renew and generate a smaller daughter cell, and in this process they express a number of key cell fate determinants and segregate these to the smaller daughter cell where they act in the specification of this cell's fate [55-57]. The progenitor cell types found in Type I and Type II lineages manifest different combinations of these cell fate determinants and 
grasshopper

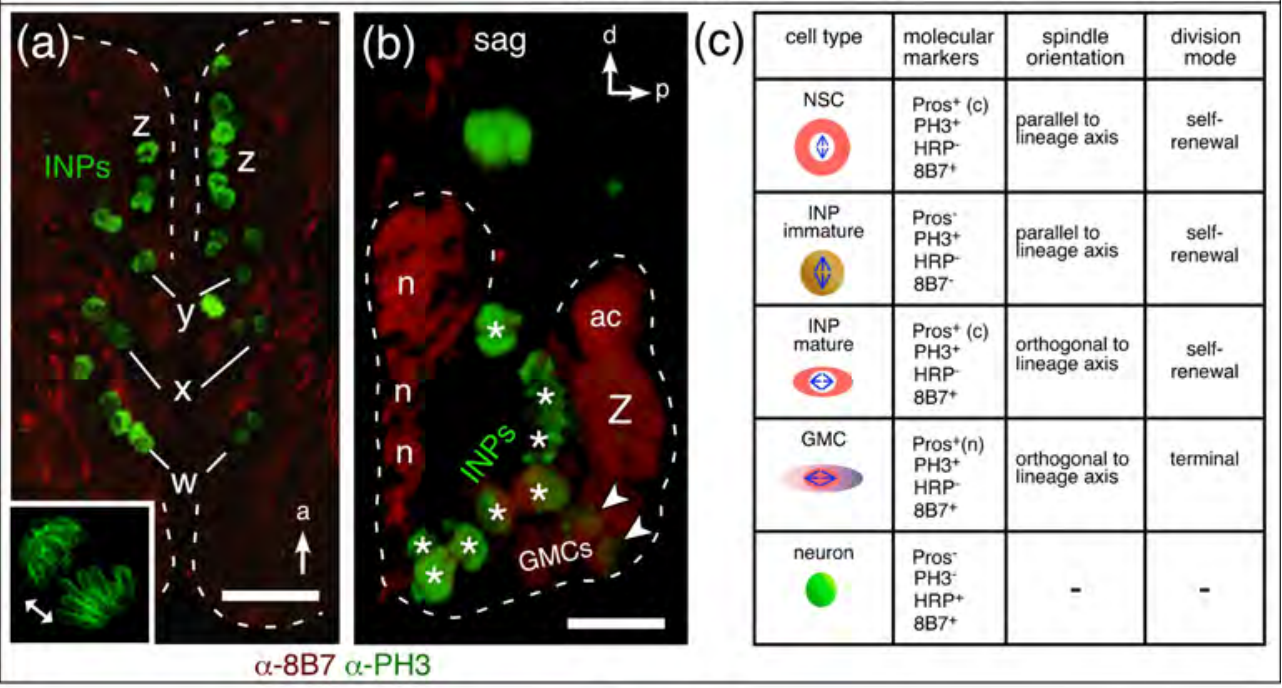

\section{Drosophila}
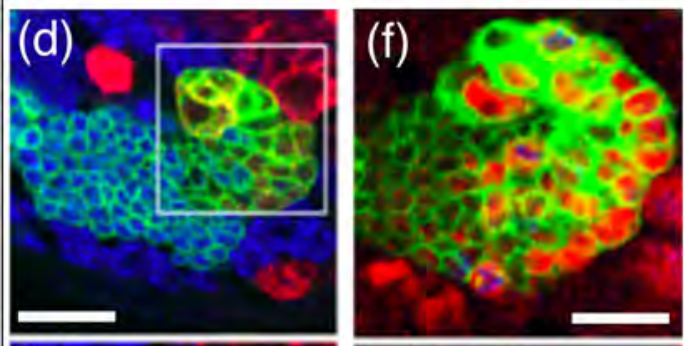

(h)

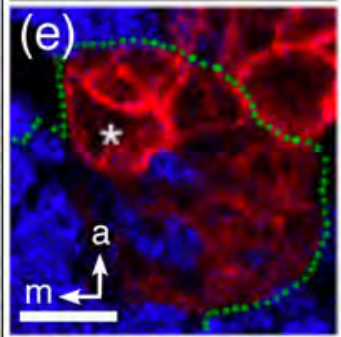

CD8::GFP Elav Mira
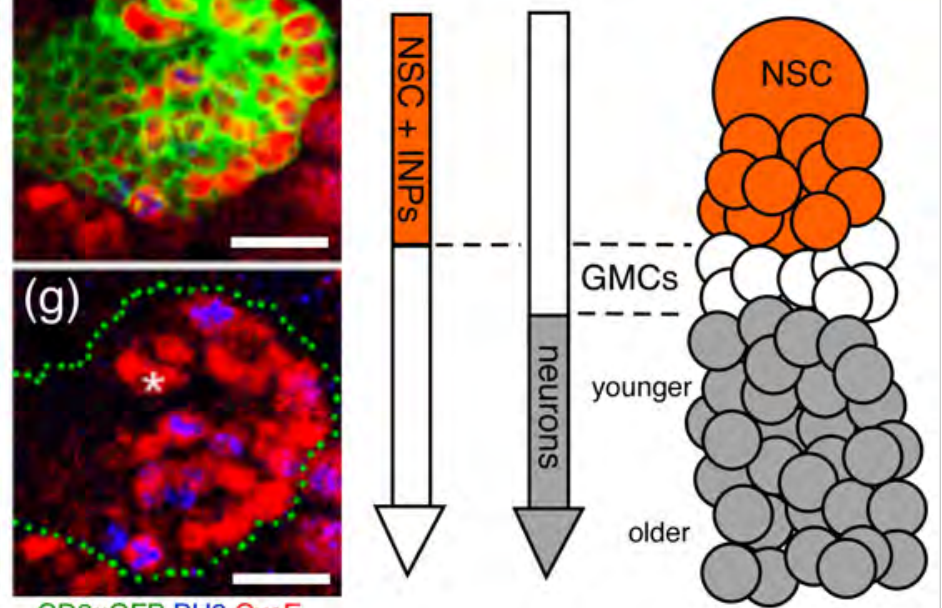

CD8::GFP PH3 CycE 
other key developmental control genes and, hence, can be identified on the basis of their molecular expression patterns. For example, all Type I NSCs express both the basic helixloop-helix transcription factor Deadpan (Dpn) and the proneural transcription factor Asense (Ase), and hence, can be distinguished from all Type II NSCs which express Dpn but do not express Ase [26,28]. Similarly, immature INPs are Dpn-negative and Asenegative and thus can be distinguished from mature INPs which are Dpn-positive and Asepositive $[26,28]$.

Three key asymmetrically segregated cell fate determinants expressed in Type I NSCs are Numb, a tissue-specific repressor of the Notch pathway, Brain tumor (Brat), a translational regulator and cell growth inhibitor, and Prospero (Pros), a transcriptional activator/repressor (for reviews, see $[28,53,58]$ ). In Type I NSCs, all three cell fate determinants act to ensure that the smaller daughter cell adopts a GMC fate and undergoes a single terminal division. In Type II NSCs, Numb and Brat are also expressed and asymmetrically segregated to the smaller daughter cell, however, Prospero is not [25-28]. As a consequence, the smaller daughter cell (the new-born, immature INP) that derives from the Type II NSCs does not receive the Prospero cell fate determinant [25-27]. Since the Prospero transcription factor has the dual function of repressing genes required for self-renewal, such as stem cell fate genes and cell cycle genes, as well as activating genes for terminal differentiation [59], the absence of Prospero in the new-born INP daughter confers stem-cell like properties to this intermediate progenitor allowing it to undergo a limited number of self-renewing proliferative divisions (Fig. 4a). Unlimited self-renewal and proliferation of mature INPs is prevented by the expression of the dFezf/Earmuff transcription factor which functions cell-autonomously to maintain the restricted mitotic potential of these cells [54].

\section{Conclusions and perspectives}


(a)

Drosophila

Type I lineage

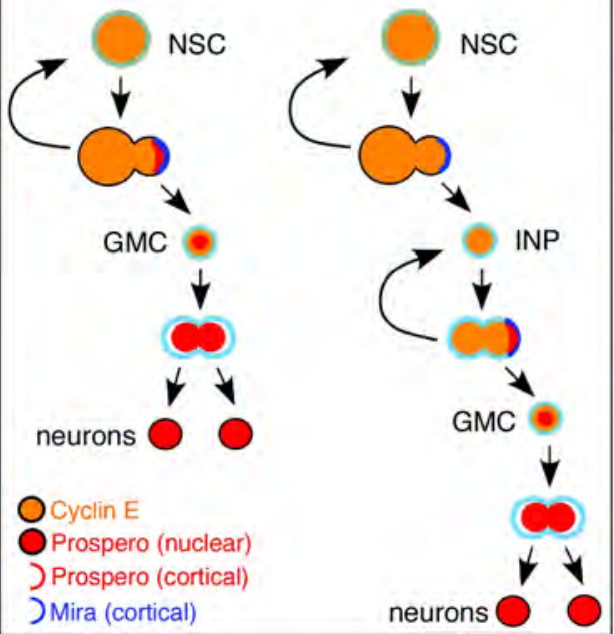

Type II lineage (b) mammal

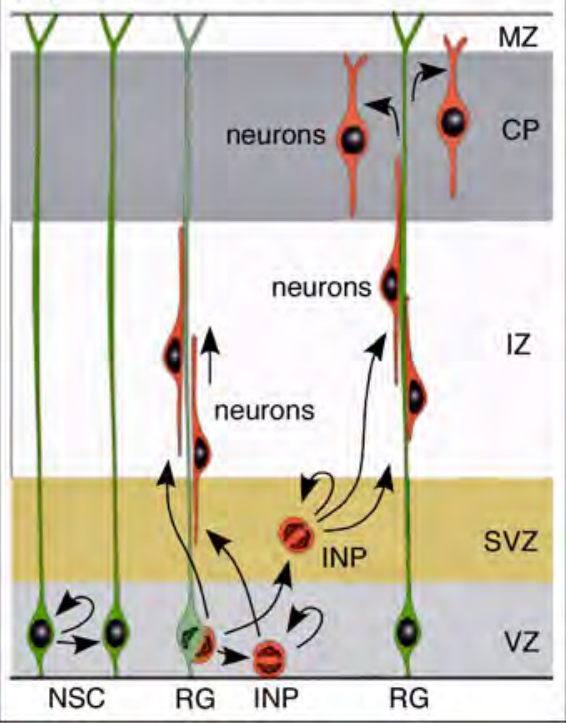


The amplification of neural proliferation through self-renewing intermediate progenitor cells may be a general mechanism for generating complexity in the developing brains of other animals, including mammals. While some of the neurons of the mammalian brain derive directly from the division of NSCs, many other neurons (as well as glial cells), notably in the developing mammalian cortex, are generated indirectly by NSCs through one or multiple rounds of divisions of INPs, also referred to as basal progenitors, with more restricted potential (Fig. 4b; see [39] for a review). Thus, in mammalian cortical development, as in insect central complex development, intermediate progenitor cells, that are functionally analogous to the transit amplifying cells present in the stem cell lineages in other tissues, play a central role in the amplification of proliferation required to generate the enormous number of neural cells that make up complex neural circuitry [60,61]. Since, to our knowledge, self-renewing INP cells have only been reported in insects and mammals, it will now be important to determine if INP-dependent amplification of NSC proliferation occurs in other animal taxa and, hence, if it is a general strategy for increasing size and complexity of the brain during development and evolution (see Box 3).

Although the amplification of NSC proliferation via INPs in Type II lineages leads to the large numbers of neurons required in central complex development, this type of amplification is also prone to proliferative dysregulation and can lead to brain tumor formation if genes involved in asymmetric cell fate determination are defective (Box 2). Thus, the neural progenitors in the insect brain that are involved in a type of INPdependent amplifying mode of neurogenesis comparable to that seen in the mammalian brain also appear to be the most highly prone to tumorigenesis. Understanding the molecular and developmental pathways that go awry and lead to uncontrolled proliferation of these cell populations may lead to important insights for future therapeutic treatments for brain tumors. 


\section{Acknowledgements}

This work was supported by Deutsche Forschungsgemeinschaft grant BO 1434/3-5 to GSB and by Swiss National Science Foundation grant 31003A124888/1 and National Research Program 63 (Stem cells and regenerative medicine) grant 406340128006/1 to HR.

Disclosure Statement: The authors declare that they have no conflict of interest related to this manuscript 


\section{Reference list}

1 Strausfeld, N.J. (1976) Atlas of an insect brain. Springer, Berlin Heidelberg New York

2 Strausfeld, N.J. (2009) Brain organization and the origin of insects: an assessment. Proc. R. Soc. B 276, 1929-1937

3 Williams, J.L.D. (1975) Anatomical studies of the insect central nervous system: a ground-plan of the midbrain and an introduction to the central complex in the locust, Schistocerca gregaria (Orthoptera). J. Zool. Lond. 176, 67-86

4 Homberg, U. (1987) Structure and functions of the central complex in insects. In: Gupta AP (ed) Arthropod brain: Its evolution, development, structure, and functions. Wiley Press, New York, pp 347-367

5 Hanesch, U. et al. (1989) Neuronal architecture of the central complex in Drosophila melanogaster. Cell Tiss. Res. 257, 343-366

6 Wegerhoff, R. et al. (1996) Development of locustatachykinin immunopositive neurons in the central complex of the beetle Tenebrio molitor. J. Comp. Neurol. 375, 157-166

7 Loesel, R., et al. (2002) Common design in a unique midline neuropil in the brains of arthropods. Arth. Struct. Dev. 31, 77-91

8 Heinze, S. and Homberg, U. (2008) Neuroarchitecture of the central complex of the desert locust: intrinsic and columnar neurons. J. Comp. Neurol. 511, 454-478

9 El Jundi, B. et al. (2010) The locust standard brain: a 3D standard of the central complex as a platform for neural network analysis. Front. Sys. Neurosci. 3, 21.

10 Homberg, U. (1994) Flight-correlated activity changes in neurons of the lateral accessory lobes in the brain of the locust Schistocerca gregaria. J. Comp. Physiol. A 175, $597-610$

11 Strauss, R. and Heisenberg, M. (1993) A higher control center of locomotor behavior in the Drosophila brain. J. Neurosci. 13, 1852-1861 
12 Strauss, R. (2002) The central complex and the genetic dissection of locomotor behaviour. Curr. Opin. Neurobiol. 12, 633-638

13 Mappes, M. and Homberg, U. (2004) Behavioural analysis of polarisation vision in tethered flying locusts. J. Comp. Physiol. A 190, 61-68

14 Liu, G. et al. (2006) Distinct memory traces for two visual features in the Drosophila brain. Nature 439, 551-556

15 Heinze, S. and Homberg, U. (2007) Maplike representation of celestial e-vector orientations in the brain of an insect. Science 315, 995-997

16 Neuser, K. et al. (2008) Analysis of a spatial orientation memory in Drosophila. Nature $453,1244-1247$

17 Weinrich, A. et al. (2008) Suppression of grasshopper sound production by nitric oxidereleasing neurons of the central complex. J. Comp. Physiol. A 194, 763-76

18 Pan, Y. et al. (2009) Differential roles of the fan-shaped body and the ellipsoid body in Drosophila visual pattern memory. Learn. Mem. 16, 289-295

19 Ilius, M. et al. (1994) The central complex of Drosophila melanogaster is involved in flight control: Studies on mutants and mosaics of the gene ellipsoid body open. $J$. Neurogenet. 9, 189-206

20 Williams, J.L.D. and Boyan, G.S. (2008) Building the central complex of the grasshopper Schistocerca gregaria: axons pioneering the w, x, y, z tracts project onto the primary commissural fascicle of the brain. Arthr. Struct. Dev. 37, 129-140

21 Ito, K. and Awasaki, T. (2008) Clonal unit architecture of the adult fly brain. In: Technau GM (ed) Brain Development in Drosophila melanogaster. Springer, New York, pp 137-158.

22 Izergina, N. et al. (2009) Postembryonic development of transit amplifying neuroblast lineages in the Drosophila brain. Neural Dev. 4, 44 
23 Bayraktar, O.A. et al. (2010) Drosophila type II neuroblast lineages keep Prospero levels low to generate large clones that contribute to the adult brain central complex. Neural Dev. 5, 26.

24 Pereanu, W. et al. (2010) A lineage-based analysis of the development of the central complex of the Drosophila brain. J. Comp. Neurol. 519:661-89

25 Bello, B.C. et al. (2008) Amplification of neural stem cell proliferation by intermediate progenitor cells in Drosophila brain development. Neural Dev. 3, 5

26 Boone, J.Q. and Doe, C.Q. (2008) Identification of Drosophila type II neuroblast lineages containing transit amplifying ganglion mother cells. Dev. Neurobiol. 68, 11851195

27 Bowman, S.K. et al. (2008) The tumor suppressors Brat and Numb regulate transitamplifying neuroblast lineages in Drosophila. Dev. Cell 14, 535-546

28 Doe, C.Q. (2008) Neural stem cells: balancing self-renewal with differentiation. Development 135, 1575-1587

29 Boyan, G.S. et al. (2010) Proliferative cell types in embryonic lineages of the central complex of the grasshopper Schistocerca gregaria. Cell Tiss. Res. 341, 259-277

30 Reichert, H. and Boyan, G.S. (1997) Building a Brain: Insights from Insects. Trends Neurosci. 20, 258-264

31 Zacharias, D. et al. (1993) Neurogenesis in the insect brain: Cellular identification and molecular characterization of brain neuroblasts in the grasshopper embryo. Development 118, 941-955

32 Younossi-Hartenstein, A. et al. 1996. Early neurogenesis in the Drosophila brain. $J$. Comp. Neurol. 370, 313-329

33 Younossi-Hartenstein, A. et al. 1997. Control of early neurogenesis of the Drosophila brain by the head gap genes tll, otd, ems and btd. Dev. Biol. 182, 270-283

34 Boyan, G.S. and Williams, J.L.D. (1997) Embryonic development of the pars intercerebralis/central complex of the grasshopper. Dev. Genes Evol. 207, 317-329 
35 Urbach, R. and Technau, G.M. (2003) Early steps in bulding the insect brain: neuroblast formation and segmental patterning in the developing brain of different insect species. Arthr. Struct. Dev. 32, 103-123

36 Urbach, R. et al. (2003a) The pattern of neuroblast formation, mitotic domains, and proneural gene expression during early brain development in Drosophila. Development $130,3589-3606$

37 Urbach, R. et al. (2003b) Spatial and temporal pattern of neuroblasts, proliferation, and Engrailed expression during early brain development in Tenebrio molitor L. (Coleoptera). Arthr. Struct. Dev. 32, 125-140

38 Williams, J.L.D. et al. (2005) Building the central complex of the grasshopper Schistocerca gregaria: temporal topology organizes the neuroarchitecture of the w, x, y, z tracts. Arthr. Struct. Dev. 34, 97-110

39 Kriegstein, A. and Alvarez-Buylla, A. (2009) The glial nature of embryonic and adult neural stem cells. Ann. Rev. Neurosci. 32, 149-184

40 Hartenstein, V. et al. (2008) The development of the Drosophila larval brain. Adv. Exp. Med. Biol. 628, 1-31

41 Skeath, J.B. and Thor, S. (2003) Genetic control of Drosophila nerve cord development. Curr. Opin. Neurobiol. 13, 8-15

42 Urbach, R. and Technau, G.M. (2004) Neuroblast formation and patterning during early brain development in Drosophila. Bioessays 26, 739-751

43 Technau, G.M. et al. (2006) Generation of cell diversity and segmental pattern in the embryonic central nervous system of Drosophila. Dev. Dyn. 235, 861-869

44 Kao, C.F. and Lee, T. (2010) Birth time/order-dependent neuron type specification. Curr. Opin. Neurobiol. 20, 14-21

45 Bullock, T.H. and Horridge, G.A. (1965) Structure and function in the nervous systems of invertebrates. Two Volumes. Freeman, San Francisco 
46 Strausfeld, N.J. (1998) Crustacean-insect relationships: the use of brain characters to derive phylogeny amongst segmented invertebrates. Brain Behav. Evol. 52, 186-206

47 Renn, S.C.N. et al. (1999) Genetic analysis of the Drosophila ellipsoid body neuropil: organization and development of the central complex. J. Neurobiol. 41, 189-207

48 Young, J.M. and Armstrong, J.D. (2010a) Structure of the adult central complex in Drosophila: organization of distinct neuronal subsets. J. Comp. Neurol. 518, 1500-1524

49 Young, J.M. and Armstrong, J.D. (2010b) Building the central complex in Drosophila: the generation and development of distinct subsets. J. Comp. Neurol. 518, 1525-1541

50 Lee, T. and Luo, L. (1999) Mosaic analysis with a repressible cell marker for studies of gene function in neuronal morphogenesis. Neuron 22, 451-461

51 Cayre, M. et al. (1994) Neurogenesis in an adult insect brain and its hormonal control. Nature 368, 57-59

52 Goodman, C.S. and Doe, C.Q. (1994) Embryonic development of the Drosophila central nervous system. In: Bate, M., Martinez-Arias, A. (Eds.), The Development of Drosophila. Vol 1. Cold Spring Harbor Press, New York, pp 1131-1206

53 Knoblich, J.A. (2008) Mechanisms of asymmetric stem cell division. Cell 132, 583-597

54 Weng, M. and Lee, C.Y. (2010) Keeping neural progenitor cells on a short leash during Drosophila neurogenesis. Curr. Opin. Neurobiol. DOI:10.1016/j.conb.2010.09.005

$55 \mathrm{Yu}$, F. et al. (2006) Drosophila neuroblast asymmetric cell division: Recent advances and implications for stem cell biology. Neuron 51, 13-20

56 Egger, B. et al. (2008) Insights into neural stem cell biology from flies. Phil Trans $R$ Soc Lond B Biol Sci 363, 39-56

57 Zhong, W. and Chia, W. (2008) Neurogenesis and asymmetric cell division. Curr. Opin. Neurobiol. 18, 4-11

58 Neumüller, R.A. and Knoblich, J.A. (2009) Dividing cellular asymmetry: asymmetric cell division and its implications for stem cells and cancer. Genes Dev. 23, 2675-2699 
59 Choksi, S.P. et al. (2006) Prospero acts as a binary switch between self-renewal and differentiation in Drosophila neural stem cells. Dev. Cell 11, 775-789

6o Noctor, S.C. et al. (2007) Contribution of intermediate progenitor cells to cortical histogenesis. Arch. Neurol. 64, 639-642

61 Miyata, T. et al. (2010) Mechanisms that regulate the number of neurons during mouse neocortical development. Curr. Opin. Neurobiol. 20, 22-28

62 Gonzalez, C. (2007) Spindle orientation, asymmetric division and tumour suppression in Drosophila stem cells. Nat. Rev. Genet. 8, 462-472

63 Januschke, J. and Gonzalez, C. (2008) Drosophila asymmetric division, polarity and cancer. Oncogene 27, 6994-7002

64 Bello, B. et al. (2006) The brain tumor gene negatively regulates neural progenitor cell proliferation in the larval central brain of Drosophila. Development 133, 2639-2648

65 Betschinger, J. et al. (2006) Asymmetric segregation of the tumor suppressor Brat regulates self-renewal in Drosophila neural stem cells. Cell 124, 1241-1253

66 Lee, C.Y. et al. (2006) Brat is a Miranda cargo protein that promotes neuronal differentiation and inhibits neuroblast self-renewal. Dev. Cell 10, 441-449

67 Wang, H. et al. (2006) Aurora-A acts as a tumor suppressor and regulates self-renewal of Drosophila neuroblasts. Genes Dev. 20, 3453-3463

68 Caussinus, E. and Gonzalez, C. (2005) Induction of tumor growth by altered stem-cell asymmetric division in Drosophila melanogaster. Nat. Genet. 37, 1125-1129

69 Kurylas, A.E. et al. (2008) Standardized atlas of the brain of the desert locust, Schistocerca gregaria. Cell Tiss. Res. 333, 125-145

70 Nässel, D.R. (1993) Neuropeptides in the insect brain: a review. Cell Tiss. Res. 273, 1-29

71 Rakic, P. (2007) The radial edifice of cortical architecture: from neuronal silhouettes to genetic engineering. Brain Res. Rev. 55, 204-219 
72 Karlstrom, R.O. et al. (1993) Lachesin: an immunoglobulin superfamily protein whose expression correlates with neurogenesis in grasshopper embryos. Development 118, 509-522

73 Boyan, G.S. et al. (2008) An ontogenetic analysis of locustatachykinin-like expression in the central complex of the grasshopper Schistocerca gregaria. Arthr. Struct. Dev. 37, $480-491$

74 Takizawa, T. and Meshorer, E. (2008) Chromatin and nuclear architecture in the nervous system. Trends Neurosci 31, 343-352

75 Jan, L.Y. and Jan, Y.N. (1982) Antibodies to horseradish-peroxidase as specific neuronal markers in Drosophila and grasshopper embryos. Proc. Natl. Acad. Sci. USA $79,2700-2704$

76 Seeger, M. et al. (1993) Mutations affecting growth cone guidance in Drosophila genes necessary for guidance towards or away from the midline. Neuron 10, 409-426

77 Halter, D.A. et al. (1995) The homeobox gene repo is required for the differentiation and maintenance of glial function in the embryonic nervous system of Drosophila melanogaster. Development 121, 317-322 


\section{Figure legends}

Figure 1: NSCs of the central complex in grasshopper (a-f) and Drosophila (g,h).

a. Map of NSCs from each protocerebral hemisphere (PC) of the grasshopper as at midembryogenesis. NSCs occupy stereotypic locations which are maintained until the cells undergo apoptosis towards the end of embryogenesis. Of the 96 NSCs present in each PC, 8 from the so-called pars interecerbralis (PI) region (denoted as black cells) generate progeny which build the modules of the central complex. Progeny from four (termed W, X, $\mathrm{Y}, \mathrm{Z}$ ) contribute fiber projections to the central body itself via the $\mathrm{w}, \mathrm{x}, \mathrm{y}, \mathrm{z}$ tracts (see Box 1 Figure Ib). Other abbreviations: OL optic lobe. Anterior (a) is to the top. Panel modified, with permission, from [31]. b. Photomicrograph of a central complex lineage of the grasshopper during very early embryogenesis following intracellular injection of lucifer yellow dye into a NSC from a living brain. Dye has spread from the NSC to two GMCs (black stars) and four progeny (black asterisks). Panel modified, with permission from [38]. c-e. Development of the lineage generating the $\mathrm{z}$ tract of the central complex, derived from the Z NSC (shaded blue). Arrows point to the posterior (p) and dorsal (d) orientations and apply to all three panels. Data modified, with permission, from [73]. (c) At a stage when $31 \%$ of embryogenesis has been completed, the Z NSC has generated about 15 progeny, none of which have yet pioneered the $\mathrm{z}$ tract. (d) At 39\% of embryogenesis, the NSC has generated over 50 progeny and the $\mathrm{z}$ tract is now visible (shaded grey), but contains less than 10 fibres. (e) At a stage when $50 \%$ of embryogenesishas been completed, there are over 100 progeny in the cluster. Cells which have been identified as making an axonal contribution to the $\mathrm{z}$ tract are shaded grey. f. Drawing of the grasshopper brain in side view (only inset to scale) showing the location in the protocerebrum of the $\mathrm{Z}$ cluster at $100 \%$ of embryogenesis. The lineage contains up to 150 progeny whose age progresses in the direction of the dashed grey line. Axons project via the $\mathrm{z}$ tract to the protocerebral 
bridge (PB). Oldest cells (black) are located at the distal tip of the lineage. Inset shows a magnified view of the most distal part of the $\mathrm{Z}$ cluster. The axon projections of 14 identified cells (black) into the $\mathrm{z}$ tract reveal a temporal topology [73]. Other abbreviations: DC, deutocerebrum; TC, tritocerebrum; $\mathrm{VNC}$, ventral nerve cord; a,b,c, indicate subsets of neuronal somata within the lineage with topographically ordered projections into the associated tract. Panel modified, with permission, from [38]. g. Confocal image shows green fluorescent protein (GFP)-labelled (green) Dorsomedial (DM) protocerebral lineages of the larval brain of Drosophila which contribute to the central complex. White arrowheads point to the four DM lineages (DM1-4) that correspond to the $\mathrm{w}, \mathrm{x}, \mathrm{y}, \mathrm{z}$ lineages of the grasshopper brain shown in (a). Also shown are glial cells of the brain (magenta, immunolabeled for the glial specific homeobox protein Reversed polarity, Repo). Anterior (a) is to the top, and the midline (m) is indicated. Panel modified, with permission from [22]. h. Confocal image showing a MARCM clone with its NSC in the protocerebrum of the larval brain of Drosophila. Cells in the cluster are GFP-labeled (green) and express the neuronal marker Embyronic Lethal Abnormal Vision (ELAV) (blue). Other markers: cell cycle marker Cyclin E (red). Panel modified, with permission, from [2]. Scale bars represent 70 $\mu \mathrm{m}(\mathrm{a}), 40 \mu \mathrm{m}(\mathrm{b}), 25 \mu \mathrm{m}$ (c-e), $20 \mu \mathrm{m}$ for magnified view in (f), $15 \mu \mathrm{m}$ in (g) and $7 \mu \mathrm{m}$ in (h).

Figure 2: Structure-specific neural stem cell lineages in the brains of grasshopper (a-c) and Drosophila (d-h).

a. Reconstructions show that protocerebral Type I lineages from the grasshopper brain (not involved with the central complex) typically comprise around 20 cells at midembryogenesis: an NSC (black star), several GMCs (black asterisks) and fifteen or so progeny. b. Confocal image of a single optical section through the bilaterally symmetrical 
Type II z lineages (brain midline, $\mathrm{m}$ ) following anti-1C10 labeling at a stage at which $50 \%$ of embryogenesis has been completed. Early in development the anti-1C10 antibody labels a cell surface moiety of the Ig superfamily expressed by cells involved in neurogenesis such as NSCs and GMCs, and later, their progeny as well [72]. The locations of the NSC (Z) and GMCs (white asterisks) in each lineage are indicated, as well as the approximate lineage extent (dashed white). An arrow points to the dorsal (d) orientation. c. Reconstructions of two Type II lineages $(\mathrm{z}, \mathrm{w})$ late in embryogenesis and whose progeny contribute projections via the protocerebral bridge $(\mathrm{PB})$ to the $\mathrm{z}$ and $\mathrm{w}$ tracts of the central complex in the grasshopper. Drawings are from horizontal serial sections following labeling with antihorseradish peroxidase (HRP) which recognizes a neuron-specific cell surface glycoprotein [75]. 120 or more progeny are present in such lineages. NSCs have undergone apoptosis by this stage; the position they occupied during development is therefore indicated schematically. Anterior (a) is to the top, medial (m) to the right. Panels (a-c) modified, with permission, from [29]. d,e. Type I and Type II NSC lineages respectively; MARCM clones (GFP-labeled, green) in the larval brain of Drosophila. Progenitor cells of the brain express Miranda (purple) so that GFP-labeled NSCs appear white (white arrowheads). Type II lineages comprise an average of 450 adult-specific cells (range 370-580), more than three times the average number of cells found in Type I lineages. Anterior (a) is to the top, medial (m) to the right. Panels (d, e) modified, with permission, from [25]. f, g. Consecutive confocal images (from dorsal to ventral) of a MARCM-labeled DM clone (GFP, green). Neuronal cells are ELAV-positive (red) and glial cells are Repo-positive (blue, white arrowheads). h. Scheme of the DM lineage; localization of the glial cells (blue, white arrowhead) is distal to the NSC in the clone. Panels (f-h) modified, with permission, from [22]. Scale bar represents $30 \mu \mathrm{m}$ in a, $50 \mu \mathrm{m}$ in b, $45 \mu \mathrm{m}$ in c, $50 \mu \mathrm{m}$ in $\mathrm{d}$ and e, $15 \mu \mathrm{m}$ in $\mathrm{f}-\mathrm{h}$.

Figure 3: INPs in the brain of embryonic grasshopper (a-c) and larval Drosophila (d-h). 
a. Confocal image of a horizontal brain slice from the grasshopper at mid-embryogenesis stained with anti-phosphohistone $3\left(\mathrm{PH}_{3}\right)$ which labels one of the four core histones wrapped inside genomic DNA and can thus be used to detect dividing cells [74]. $\mathrm{PH}_{3}-$ immunoreactive INPs (green) arrayed within the $w, x, y, z$ lineages of each brain hemisphere (outlined dashed white) are illustrated. Note bilateral symmetry. The INPs in each array maintain their organization and number (5-7 per lineage) for up to a fifth of embryogenesis. Remaining brain neuroarchitecture is immunoreactive for 8B7 (red). The monoclonal antibody $8 \mathrm{~B} 7$ recognizes the Akt2 isoform of protein kinase B and is expressed early in development in NSCs and their progeny, later in axonal projections [76]. Inset shows a high power confocal view of the chromosomal organization within an individual INP captured at meta/anaphase of the cell cycle via $\mathrm{PH}_{3}$ immunohistochemistry. Arrow indicates spindle axis (always parallel to the lineage axis). Anterior (a) is to the top. b. Confocal image of the $\mathrm{z}$ lineage in sagittal (sag) view at mid-embryogenesis shows $8 \mathrm{~B} 7$ immunoreactive accessory cell (ac), NSC (Z), GMCs and neuronal progeny (n) (red) of the lineage along with the attendant array of $\mathrm{PH}_{3}$ immunoreactive INPs (green, white asterisks). One of the GMCs (white arrowheads) is mitotically active. Arrows point to dorsal (d), posterior (p). c. Table showing identifying characteristics for cell types within Type II lineages associated with the central complex of the grasshopper brain. Molecular markers, spindle orientation and division mode are compared between the different cell types. Abbreviations: Pros n, nuclear Prospero expression; Pros c, cortical Prospero expression; PH3, HRP. Panels a,b,c modified, with permission, from [29]. d-g. Confocal images of DM (Type II) lineages in the larval brain of Drosophila MARCM-labeled with GFP (green) and immunostained with the markers indicated. (The GFP channel is omitted for clarity in the lower panels and green dots outline the clones.). d, e. Progenitor cells in a lineage include the NSC identifiable by its size (asterisk) and the most recently born INP which are found in close spatial proximity to the NSC and are characterized by a weak level of cortical Miranda (red) and the absence of the neuronal marker ELAV (blue). A close up 
view of the area boxed in (d) is shown in (e). f, g. Proliferating progenitor cells in the lineage express the cell cycle markers Cyclin E (red) and/or $\mathrm{PH}_{3}$ (blue). h. Schematic organization of cell types and temporal topology in DM (Type II) lineages. DM NSCs are associated with a cluster of proliferating INPs (orange). Neuronal progeny (gray) are ELAV-positive and located distally in the lineage. Panels d,e,f,g,h modified, with permission, from Ref. [25]. Scale bar represents $40 \mu \mathrm{m}$ in a, $8 \mu \mathrm{m}$ in inset; $25 \mu \mathrm{m}$ in b, $12 \mu \mathrm{m}$ in $\mathrm{d}, 7 \mu \mathrm{m}$ in $\mathrm{e}, 8 \mu \mathrm{m}$ in $\mathrm{f}, \mathrm{g}$.

Figure 4: Mechanisms for increased complexity in brain lineages of Drosophila and mammal.

a. Type I and Type II lineages in Drosophila. In Type I (classical) lineages, the NSC divides asymmetrically to self-renew and generate a GMC. A single terminal division of the GMC then produces two neural progeny. Unequal partitioning of Prospero and Miranda (Mira) from the NSC to the GMC promotes this terminal neurogenic division by inhibiting selfrenewal in the GMC. In Type II (DM) lineages associated with the central complex, the NSC divides asymmetrically to self-renew and generate an INP without Prospero, which enables the INP to retain a limited self-renewing potential. The INP divides to self-renews and generate a GMC which undergoes a single terminal division to produce two neural progeny. In self-renewing INP, Prospero and other cell fate determinants are reestablished and asymmetrically segregated to the GMC daughter to its promote terminal division. This amplifying mode of neurogenesis increases the number of neural cells that result from a single NSC division. Panel modified, with permission, from [25]. b. In the mammalian cortex, NSCs (dark green) of the ventricular zone (VZ) can increase their number via self-renewal through symmetric divisions. NSC as radial glia (RG, light green) can generate neurons in three ways. First, RG can generate neurons directly through asymmetric division. Second, RG can generate neurons indirectly via (non self-renewing) 
INPs that divide only once. Third, RG can generate neurons indirectly via self-renewing INPs that undergo multiple rounds of asymmetric proliferative divisions and hence amplify the number of neural progeny produced. INPs can generate neurons from within the VZ or migrate first into outer-more cortical layers (subventricular zone, SVZ) to generate neuronal progeny. In all cases, postmitotic neural cells then migrate dependently or independently of the radial glia into outer most cortical layers. Other abbreviations: IZ intermediate zone, $\mathrm{CP}$ cortical plate, MZ marginal zone. Panel modified with permission from [71]. 


\section{Box 1}

\section{Identified NSCs in central complex development}

The central complex is an intricate midline neuropile of the insect brain that comprises several modular subcompartments (Fig. Ia, c). Currently, a total of eight identified NSCs in each protocerebral hemisphere of the grasshopper have been shown to generate progeny contributing to the central complex. Of these, four (W, X, Y, Z) generate the columnar small field neurons (Fig. Ib). Four others also generate neurons projecting initially to associated neuropiles of the central complex [38]. In Drosophila, a total of ten identified NSCs are currently known to contribute neural progeny to the central complex [24]. As in the grasshopper, four of these located near the midline of each brain hemisphere generate the columnar small field neurons (Fig. Id). Different authors refer to these four identified lineages as the DM1-4 lineages, the FPB1-4 lineages or the DPMm1/DPMpm1/DPMpm2/CM4 lineages (21,24,25). These four NSCs as well as two other midline associated NSCs (DM5, DM6) and two NSCs (DM7, DM8) located more medially in the hemisphere exhibit the novel form of amplifying neurogenesis discussed in this review [25-27].

To date, this amplifying mode of neurogenesis has not been investigated in central complex development of other insects. However, the remarkable similarities in the developmental mechanisms underlying the generation of the central complex in a directly developing orthopteran insect such as the grasshopper and in an indirectly developing dipteran insect such as Drosophila strongly suggest that comparable mechanisms operate in central complex development of most if not all insects. Moreover, since grasshopper and fly are separated by more than 300 million years of evolutionary distance [30], it seems likely that the NSCs and their amplifying mode of neurogenesis were already operative in insect brain development before that time. Indeed, we posit that these processes may be a general and evolutionarily conserved feature of brain development in other arthropods such as crustaceans, given that unique central complex-like midline neuropiles are a 
common design feature of the brains in most arthropod taxa [2,7]. It will be important to investigate this possibility in representatives of arthropod and other invertebrate taxa that are accessible to experimental analysis of brain development. 


\section{Box 2}

\section{Proliferative dysregulation of NSCs and the formation of brain tumors}

Mutational analysis in the developing brain of Drosophila indicates that malfunction of the mechanisms that control the proliferative divisions of NSCs or the developmental fate of their daughter cell progenitors is one of the principle causes of malignant transformation of these cells. Significantly, a number of genetic studies focused on the asymmetric segregation of cell-fate determinants in NSCs have uncovered the tumor-suppressor activity of these determinants [62,63]. Prominent among these are the cell fate determinants Prospero, Brat and Numb, in that mutational loss of function for any one of the three results in dramatic primary brain tumors in Drosophila [64-67]. For example, targeted loss-of-function of the brat gene in brain NSCs results in clones of overproliferating cells in the developing brain, and these clones continue to manifest uncontrolled proliferation into adulthood resulting in massive brain tumors that are invariably lethal (Box 2 Fig. Ia-d). Moreover, transplantation of the corresponding mutant brain tissue into normal host flies results in lethal malignant tumors and metastasis formation, and the resulting tumors can be successively reimplanted into new hosts for years [68]. In terms of growth rate, cell types and metastatic activity, the transplantinduced tumors are essentially indistinguishable from one another, supporting the notion that all of these tumors might have a common etiology, namely perturbation of cell-fate determinants in NSCs.

Several lines of evidence indicate that Type II NSC lineages, such as those that normally contribute neural progeny to central complex development, may be a major source of the brain tumors which are induced by dysregulation of cell fate determinants. First, analysis of tumor phenotypes indicates that the overgrowing cells most often arise in a specific location in the dorsal brain hemispheres and this is the same region in which the amplifying Type II lineages are found $[25,64,65]$. Second, targeted loss-of-function of brat or numb specifically in Type II NSCs results in misspecified INPs that are unable to 
produce differentiated neural progeny and initiate tumorigenic overgrowth (Box 2 Fig. IIe. f). Third, molecular genetic transformation of the amplifying Type II NSCs into nonamplifying Type I NSCs appears to abolish the tumorigenic phenotype at least in brat mutants [27]. Fourth, mutational loss of the dFezf/earmuff gene responsible for limiting INP self-renewal causes a de-differentiation of INPs into ectopic Type II NSCs resulting in an uncontrolled expansion of amplifying Type II NSCs and tumorigenic overgrowth [54]. Thus, while the Type II NSCs which amplify proliferations via INPs allow differentiated neural cells to be produced in a greater number and at a faster rate than in normal nonamplifying NSC lineages, this mechanism for increased brain complexity may inherently come with a markedly higher risk of lethal brain tumor formation. 


\section{Box 3}

\section{Outstanding questions and future research directions}

- What are the origins and roles of glial cells in central complex development? This question could be experimentally addressed by the selective elimination of glial cells through targeted apoptosis. Alternatively, the formation of the central complex could be examined in mutants that lack GCMs or Repo (a protein known to be crucial for glia development) [77].

- What regulates the sequence of individual neuronal types in NSC lineages containing INPs during insect brain development? To address this question, future studies should assess temporal identity changes using high resolution single-cell lineage analysis, such as single cell MARCM, twin-spot MARCM, and/or intersectional methods for refined transgenic targeting.

- To what extent are the NSCs, their lineages, and the resulting complex brain circuitry homologous amongst insect species? Transcription factor expression, the relative position of NSCs during delamination, and common neuroanatomical properties of neural progeny should be investigated in diverse insect taxa, to add to the body of studies that currently exist for grasshopper and fruitfly.

- Are the mechanisms for building complex brain architecture phylogenetically conserved? Comparative functional analysis of conserved developmental control genes during brain development in a variety of vertebrate and invertebrate model systems should be performed to address this question.

- To what extent do the mechanisms of proliferation and proliferative dysregulation of NSCs and INPs provide general insights into brain tumorigenesis? The functional conservation of NSC/INP regulatory genes in mammalian systems should be analyzed, as well as assaying for tumor suppressor and oncogene function of these regulatory genes in mutant insect models. 
Box 1 Figure I: Neuroarchitecture of the central complex in grasshopper and fly.

a. Schematic diagram depicting the brain of the adult grasshopper (Schistocerca gregaria) viewed frontally. The central complex comprises a group of neuropilar modules in the midline of the protocerebral neuromere $(\mathrm{PB}$, protocerebral bridge; $\mathrm{CB}$, central body; $\mathrm{EB}$, ellipsoid body; LAL, lateral accessory lobes) and between the bilateral mushroom bodies (MB). Many neurons contributing to this neuroarchitecture have their cell bodies in the pars intercerebralis (PI) cortical region. Arrow indicates dorsal (d) and applies to all panels. Panel modified, with permission, from [69]. b. Drawing (semi-schematic) details the essential neuroarchitecture of the central complex in the grasshopper brain. The stereotypic eight-fold columnar organization of the central body (CB) is formed by the projections of clusters of neurons from the so-called PI of the brain midline. Each cluster derives from a separate embryonic NSC. Neuronal progeny direct fibers initially to the PB, and then to the $\mathrm{CB}$ via one of four bilaterally symmetrical tract systems (w, x, y, z). Fibers enter the CB via its dorsal chiasmal system, traverse the CB via the columnar system, and exit ventrally to run either to other modules of the central complex (ie. the EB and the LAL), or to other brain regions, or into the ventral nerve cord. A given cell cluster of the PI contains neurons expressing a range of neuromodulators (eg. serotonin, red; allatostatin I, blue; allatostatin II, green). Their projections form discrete layers in the central complex according to the biochemical identity of the cells involved. Panel modified, with permission, from [38]. c. Confocal image of a frontal section of the brain of a fly (Phormia sp.) following anti-FMRFamide (green) and anti-leucokinin (red) immunolabeling reveals layered neuroarchitecture of the $\mathrm{CB}$ and noduli $(\mathrm{N})$ in the midline of the protocerebrum. Panel modified, with permission, from [70]. d. Confocal image (horizontal plane) at 24 hrs after commencement of pupation in Drosophila melanogaster reveals four clusters of Drosophila neuronal (Dn)-cadherin labeled pontine neurons per hemisphere projecting fibers via four bundles $(1,2,3,4)$ to the $\mathrm{CB}$ of the central complex. Note the similarity to 
the cellular organization of this neuropile in the grasshopper brain depicted in panels (a,b). Panel modified, with permission, from [49]. Scale bar represents $400 \mu \mathrm{m}$ in a; $90 \mu \mathrm{m}$ in b; $55 \mu \mathrm{m}$ in c; $25 \mu \mathrm{m}$ in $\mathrm{d}$.

Box 2 Figure I Type II lineages in Drosophila and mutants of cell fate determinant genes.

a, b. Wild-type and brain tumor (brat) mutant MARCM clones labeled with GFP (white) in third instar larval brain hemispheres, counterstained with the DNA dye TOTO-3 (purple). a. Wild-type clones contain progeny of a single NSC occupying a small area of third instar larval brain (white arrowhead, single clone). b. Large brat mutant clones (white arrowhead) are difficult to resolve as single lineages (two or more merged clones are seen). c, d. brat mutant clones continue in the central to proliferate into adulthood. Wholemount adult brains showing MARCM clones which had been induced in early first instar larvae. c. Wild-type MARCM clones in central brain (arrowhead) and optic lobe (arrow) labeled with a lactose Z (lacZ) marker (blue). d. brat mutant MARCM clones in central brain (arrowheads) labeled with lacZ marker (blue) are dramatically enlarged in size. Mutant clones in optic lobe (arrows) remain wild-type-like. (Asterisks denote the remains of the head cuticle). Panels a,b,c,d modified, with permission, from [64]. e. Model of a Type II lineage in the central brain of wild-type Drosophila with a schematic of the resulting clone below. The NSC (blue) generates immature INPs which mature into selfrenewing INPs (red). These INPs generate GMCs (yellow), which divide terminally to produce two neurons (green). f. Model of a Type II lineage in the central brain of a brat mutant Drosophila with a schematic of the resulting clone below. The NSC (blue) generates mutant INPs which give rise to tumor-initiating cells that continue to proliferate 
uncontrollably. Panels e,f modified, with permission, from [27]. Scale bar represents $85 \mu \mathrm{m}$ in a,b; $90 \mu \mathrm{m}$ in c,d. 


\section{Glossary}

Asymmetric cell division: A mitotic division that produces two daughters with different cell fate such that they differ in cell size, segregated molecular constituents, or differentiation potential.

Cell fate determinant: Molecules such as Prospero, Numb and Brat which promote differentiation and the acquisition of cell identity and inhibit self-renewal during development. In neural development, cell fate determinants usually segregate from the progenitor to the differentiating cells.

Central complex: A midline neuropile structure of the insect brain involved in multimodal sensorimotor integration. It is generally considered to be comprised of five major modules: the protocerebral bridge, the central body, the ellipsoid body, the noduli and the lateral accessory lobes.

Intermediate neural progenitor (INP): A self-renewing secondary neural progenitor with limited proliferation potential that derives from NSCs, has features characteristic of transit amplifying cells, and can give rise to neuronal and/or glial progeny.

Mushroom body (MB): A neuropile structure located in each protocerebral hemisphere of the insect brain and involved in learning and memory. Each MB is comprised of a calyx, peduncle, alpha and beta lobes. So-called Kenyon cells surrounding the calyx represent the intrinsic neurons of the mushroom body.

Neural lineage: In insects, the collective progeny of an individual NSC including INPs, ganglion mother cells (GMCs), neurons and glial cells. 
Neural stem cell (NSC): A self-renewing primary progenitor cell that initiates the neural lineages ultimately leading to the formation of differentiated neuronal or glial cells. NSCs in insects are also referred to as neuroblasts.

Neuropile: A collective term for regions in the central nervous system comprising axons, dendrites, synaptic connections and glial cell processes. In insects, a neuropile is generally surrounded by a cortex of neuronal and glial cell bodies.

Mosaic analysis with a repressible cell marker (MARCM): a somatic genetic labeling technique for lineage analysis in wildtype and mutant cell clones in Drosophila.

Protocerebrum: The main, anterior-most part of the arthropod brain, which together with the deutocerebrum and tritocerebrum comprise the supraesophageal ganglion. The protocerebrum contains the central complex and mushroom bodies and is associated with the optic lobes. 


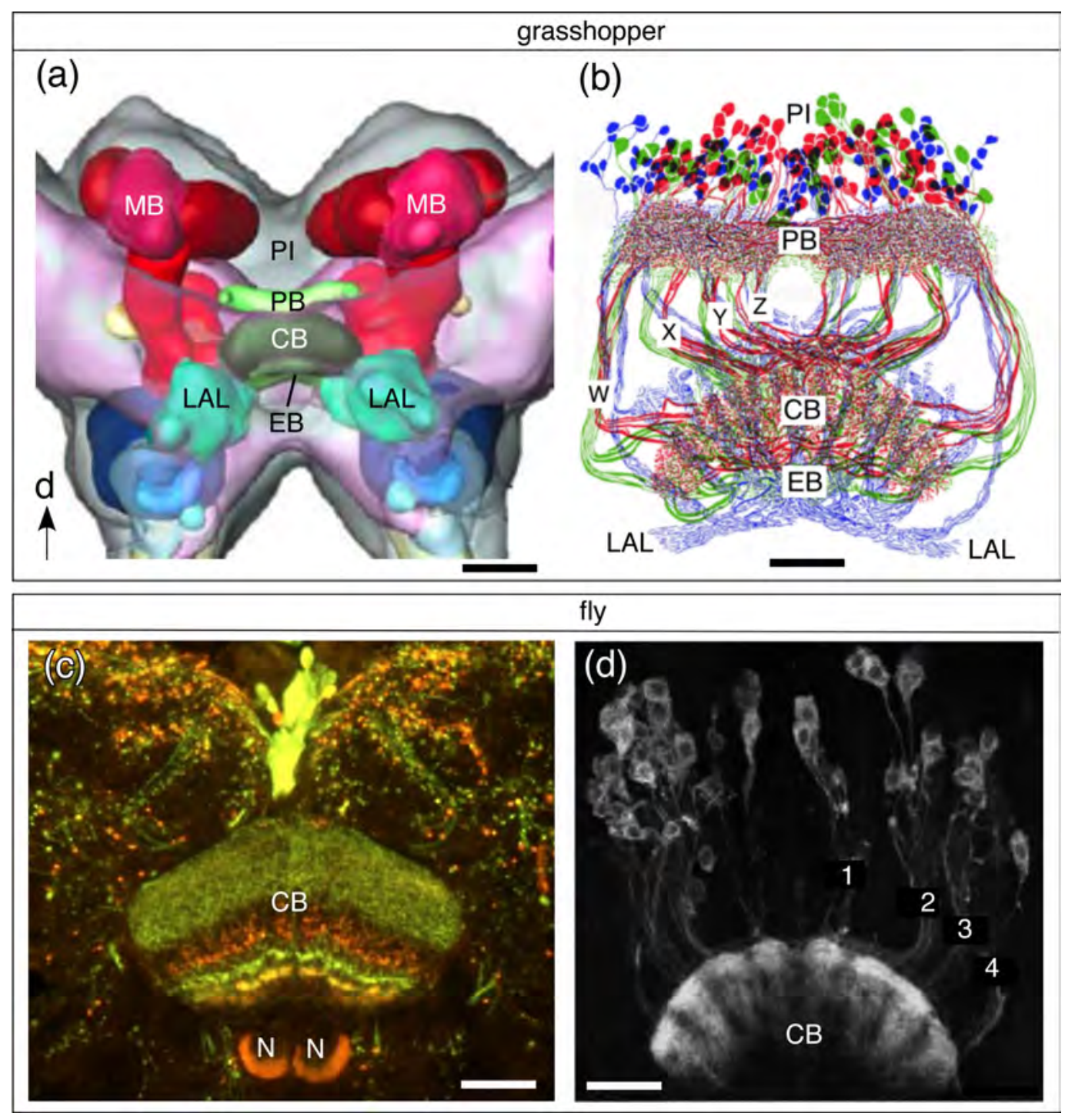



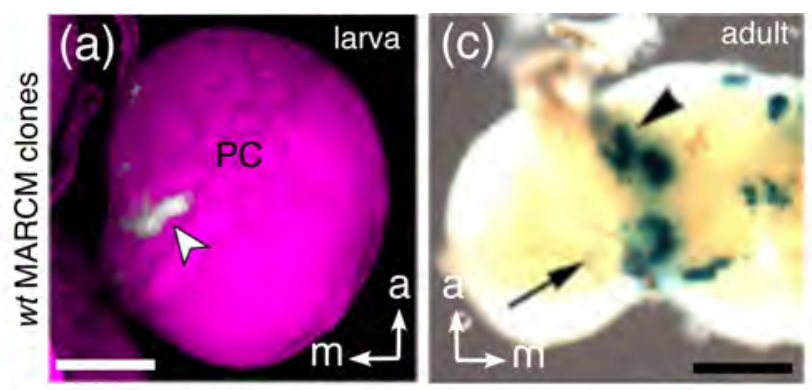

(e) Type II in WT
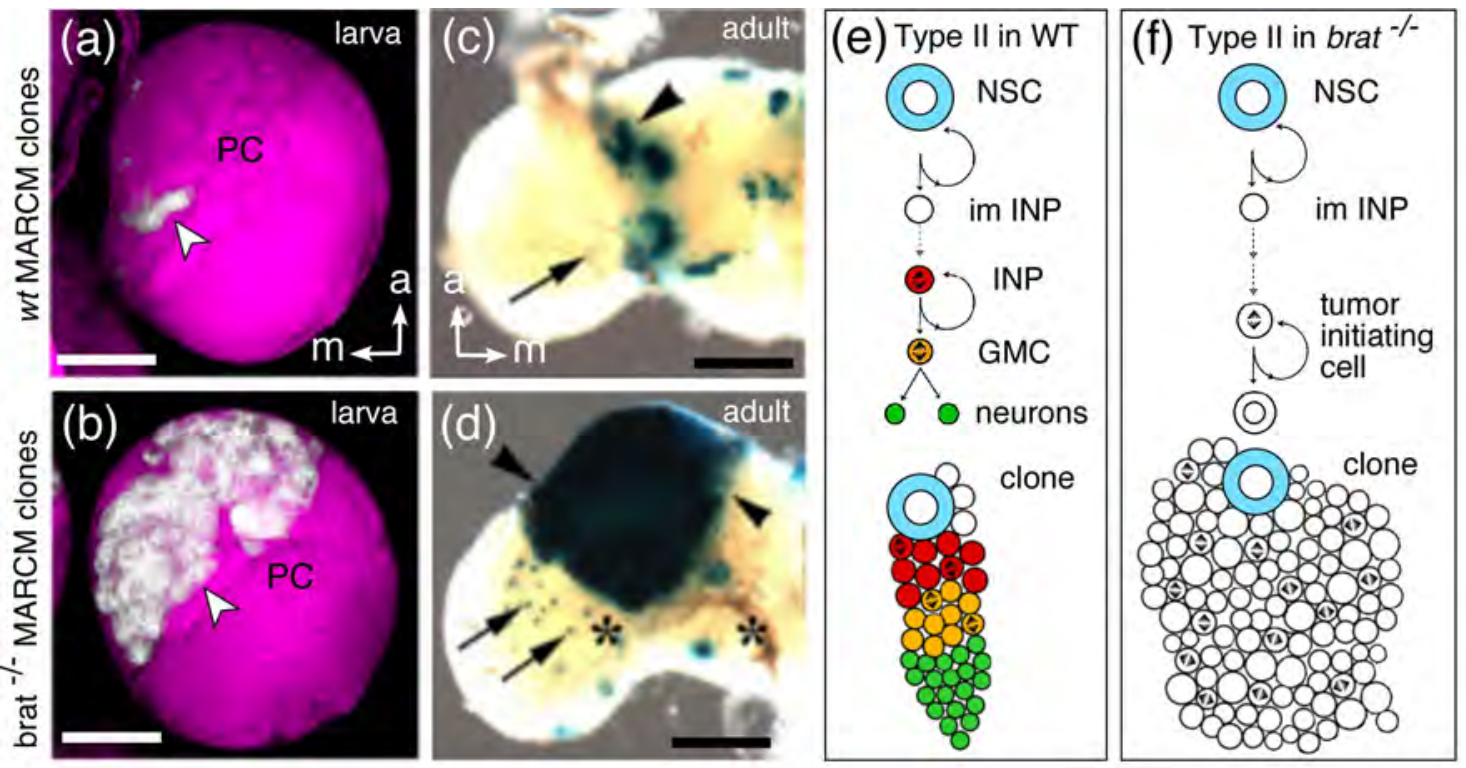

(O) $\mathrm{NSC}$

$\bigcup_{\mathrm{im} I N P}$

(j) INP

() $\mathrm{GMC}$

O neurons

OR clone

00080800 